\title{
Aplicação do nursing activities score nas unidades de terapia intensiva brasileira: revisão integrativa da literatura
}

\author{
Application of the nursing activities score in a brazilian intensive care unit: an integrative \\ literature review
}

Aplicación del nursing activities score en una unidad de cuidados intensivos de brasil: una revisión bibliográfica integradora

Sara Alves Monteiro ${ }^{1}$, Wyctória Carvalho Alves de Souza ${ }^{1}$, Lucas Alves Silva ${ }^{1}$, Nelisnelson da Silva Oliveira $^{1}$, Francisco Railson Bispo de Barros ${ }^{1 *}$.

\section{RESUMO}

Objetivo: Identificar a aplicabilidade do instrumento que mede a carga de trabalho de enfermeiros nas Unidades de Terapia Intensivas brasileiras. Métodos: trata-se de um estudo de revisão integrativa, realizado por meio de consulta as bases de dados Banco de Dados em Enfermagem (BDENF), Scientific Electronic Library Online (SCIELO), Latino-Americano e do Caribe de Informação em Ciências da Saúde (LILACS) e Medical Literatura Analysis and Retrieval (MEDLINE), no período de 2009 a 2019. Resultados: Os cruzamentos de busca nas bases de dados estabelecidas, resultaram na identificação de 44 artigos no BDENF, 71 no LILACS, 80 no PUBMED e 16 no SciELO, totalizando 241 estudos. A aplicação dos três filtros possibilitou refinamento que resultou em seleção final de 17 artigos com base na relevância e na qualidade dos dados. Observou-se que o instrumento é utilizado para quantificar e dimensionar os profissionais de enfermagem, assim como classificar a gravidade do paciente, tendo em vista os setores hospitalares considerados críticos. para a presente revisão. Considerações finais: evidenciou-se que a enfermagem intensiva brasileira tem se utilizado do Nursing Activities Score para auxilia-los na qualificação e quantificação da assistência prestada, dimensionamento da equipe de enfermagem e acompanhamento da evolução clínica do paciente em situação crítica.

Palavras-chave: Unidades de terapia intensiva, Serviços de enfermagem, Cuidados de enfermagem.

\begin{abstract}
Objective: To identify the applicability of the instrument that measures the workload of nurses in Brazilian Intensive Care Units. Methods: this is an integrative review study, carried out by consulting the databases Nursing Database (BDENF), Scientific Electronic Library Online (SCIELO), Latin American and Caribbean Health Sciences Information (LILACS) and Medical Literature Analysis and Retrieval (MEDLINE), from 2009 to 2019. Results: The search crossings in the established databases, resulted in the identification of 44 articles in BDENF, 71 in LILACS, 80 in PUBMED and 16 in SciELO, totaling 241 studies. The application of the three filters enabled refinement that resulted in a final selection of 17 articles based on the relevance and quality of the data. It was observed that the instrument is used to quantify and dimension nursing professionals, as well as to classify the severity of the patient, considering the hospital sectors considered critical. for the present review. Final considerations: it was evidenced that the Brazilian intensive nursing has been using the Nursing Activities Score to assist them in qualifying and quantifying the assistance provided, dimensioning the nursing team and monitoring the clinical evolution of the patient in a critical situation.
\end{abstract}

Keywords: Intensive care units, Nursing services, Nursing care.

${ }^{1}$ Centro Universitário do Norte (UNINORTE), Manaus - AM. *E-mail: enf.franciscobarros@gmail.com 


\section{RESUMEN}

Objetivo: identificar la aplicabilidad del instrumento que mide la carga de trabajo de las enfermeras en las unidades de cuidados intensivos de Brasil. Métodos: este es un estudio de revisión integrador, realizado a través de la consulta de las bases de datos de Enfermería (BDENF), la Biblioteca Electrónica Científica en línea (SCIELO), la Información de Ciencias de la Salud de América Latina y el Caribe. (LILACS) y Medical Literatura Analysis and Retrieval (MEDLINE), de 2009 a 2019. Resultados: Los cruces de búsqueda en las bases de datos establecidas, resultaron en la identificación de 44 artículos en BDENF, 71 en LILACS, 80 en PUBMED y 16 en SciELO, totalizando 241 estudios. La aplicación de los tres filtros permitió un refinamiento que resultó en una selección final de 17 artículos basados en la relevancia y calidad de los datos. Se observó que el instrumento se utiliza para cuantificar y dimensionar a los profesionales de enfermería, así como para clasificar la gravedad del paciente, considerando los sectores hospitalarios considerados críticos. para la presente revisión. Consideraciones finales: se evidenció que la enfermería intensiva brasileña ha estado utilizando el Puntaje de Actividades de Enfermería para ayudarlos a calificar y cuantificar la asistencia brindada, dimensionar el equipo de enfermería y monitorear la evolución clínica del paciente en una situación crítica.

Palabras clave: Unidad de cuidados intensivos, Servicios de enfermería, Cuidados de enfermería.

\section{INTRODUÇÃO}

A Enfermagem é entendida amplamente como ciência de cuidados integrais à saúde, assim assume cada vez mais um papel decisivo em relação aos cuidados prestados ao paciente com enfoque na promoção de saúde e recuperação de agravos do indivíduo, portanto constitui-se como componente fundamental dentro de um sistema de saúde (BACKES DS, et al., 2012).

Diante disso, a equipe de enfermagem está inserida na prestação de cuidados nas Unidades de Terapia Intensiva (UTI's), que são locais que assistem pessoas em situações graves ou críticas, havendo possibilidades de recuperação, ondem necessitam de maior quantidade de cuidados médicos e de enfermagem, sendo estes qualificados e de forma integral. Essas unidades possuem custo elevado para sua manutenção, por utilizar alta tecnologia, diferente espaço físico e profissional especializado (CYRINO CMS, et al., 2018).

Tendo em vista que na UTI as necessidades dos pacientes são superiores e requer maior assistência e supervisão dos profissionais, se faz necessário um número de profissionais adequados dentro da equipe de enfermagem, pois a proporção inadequada gera sobrecarga entre os profissionais, o que resulta em impactos na recuperação dos pacientes ali assistidos (GOULART LL, et al., 2017).

É um grande desafio para a assistência suprir a falta de profissionais de enfermagem, onde altas cargas de trabalho geram desgaste psicológico e fisiológico para a equipe. Não apenas é necessário conhecimento profissional para prestar um cuidado qualificado, é necessário dimensionar e obter um número de profissionais adequados para uma assistência segura e que apresente riscos mínimos aos pacientes críticos (VERSA GIGS, et al., 2011; NOVARETTI MCZ, et al., 2015).

Diante da dificuldade apresentada, o gerenciamento de enfermagem se tornou um desafio e para isso é necessário o uso de instrumentos e recursos existentes dentro da UTI, no qual é capaz de mensurar o tempo de assistência de enfermagem e dimensionamento de pessoal de acordo com as necessidades dos pacientes (BORGES F, et al., 2017).

Entre os instrumentos mais utilizados dentro da UTI estão o Time Orient Score System (TOSS), Nursing Care Recording System (NCR11), System of Patient Related Activity (SOPRA) e o Therapeutic Intervention Score (TISS), que passou a ser o atual Nursing Activities Score (NAS), se destacando por ser o mais fidedigno na avaliação da gravidade de pacientes e capaz de mensurar de maneira indireta a carga de trabalho de enfermagem (BORGES F, et al., 2017). 
O NAS surgiu com o objetivo de definir as atividades de enfermagem que melhor descrevem a carga de trabalho dos enfermeiros UTI, sendo constituído por 7 grandes categorias: atividades básicas, suporte ventilatório, cardiovascular, renal, neurológico, metabólico e intervenções específicas, totalizando 23 itens, variando de 1,2 a 32 pontos.

A aplicação do NAS tem por objetivo mensurar o tempo requerido para realização das atividades de enfermagem durante o cuidado ao paciente nas $24 \mathrm{~h}$, totalizando um escore máximo de $176,8 \%$, que representa o tempo gasto pela equipe de enfermagem na assistência ao paciente por turno (GOULART LL, et al., 2017).

Trata-se de um instrumento traduzido e validado para a realidade assistencial brasileira, de adequação quantitativa, por meio de porcentagem em tempo real gasto por um profissional de enfermagem, na assistência direta e indireta ao cliente durante 24 horas. Dessa forma, valores superiores a $100 \%$ indicam que um profissional se faz necessário para atender às necessidades de cuidados de um único paciente durante 24 horas (TRETTENE AS, et al., 2015).

Neste contexto, o presente estudo tem por objetivo identificar a aplicabilidade Nursing Activities Score em Unidades de Terapia Intensiva brasileiras. Para tanto, formulou-se a seguinte questão norteadora: A enfermagem brasileira tem utilizado o Nursing Activities Score nas Unidades de Terapia Intensiva?

\section{MÉTODOS}

A pesquisa consiste em uma revisão integrativa da literatura que objetivou compreender o estado da arte na literatura científica referente a apropriação do Nursing Activities Score (NAS) pela enfermagem intensiva brasileira de janeiro de 2019 a março de 2020.

Essa modalidade de pesquisa se caracteriza na síntese do estado dos dados científicos relacionados a um problema de pesquisa, possibilitando conclusões amplas a respeito de um tema específico, apontando possíveis lacunas que podem ser preenchidas por novas pesquisas (POLIT DF e BECK CT, 2018).

Por se tratar de uma revisão integrativa da literatura, não foi necessária a aprovação da pesquisa pelo comitê de ética em pesquisa, segundo Resolução 466/12 e 510/16 do Ministério da Saúde (BRASIL, 2012; BRASIL, 2016).

Para a realização da revisão utilizou-se o modelo proposto por Souza MT, et al. (2010), que envolve seis etapas, a saber: 1 - Elaboração da pergunta norteadora; 2 - Busca ou amostragem na literatura; 3 - Coleta de dados; 4 - Análise crítica dos estudos incluídos; 5 - Discussão dos resultados; 6 - Apresentação da revisão integrativa.

A questão que norteou esta revisão integrativa foi: "A enfermagem brasileira tem utilizado o Nursing Activities Score nas Unidades de Terapia Intensiva?"

Para a seleção dos artigos foram utilizadas as seguintes bases de dados: Banco de Dados em Enfermagem (BDENF), Latino-Americana e do Caribe de Informação em Ciências da Saúde (LILACS), Medical Literatura Analysis and Retrieval (MEDLINE/PUBMED) e Scientific Electronic Library Online (SCIELO). Os estudos encontrados em mais de uma base de dados foram considerados somente uma vez.

Para seleção dos estudos foram utilizados os seguintes critérios: publicações disponíveis na íntegra online, nos idiomas português, inglês e espanhol, estudos desenvolvidos por pesquisadores brasileiros e publicados no período compreendido entre 2009 e 2020.

Os critérios de exclusão foram: estudos em que não foi possível identificar relação com a temática por meio da leitura de título e resumo e estudos secundários. Os descritores utilizados foram: "Unidades de Terapia Intensiva" and "Serviços de Enfermagem" and "Cuidados de Enfermagem" (Figura 1). 
Figura 1 - Fluxograma da busca dos artigos da revisão.

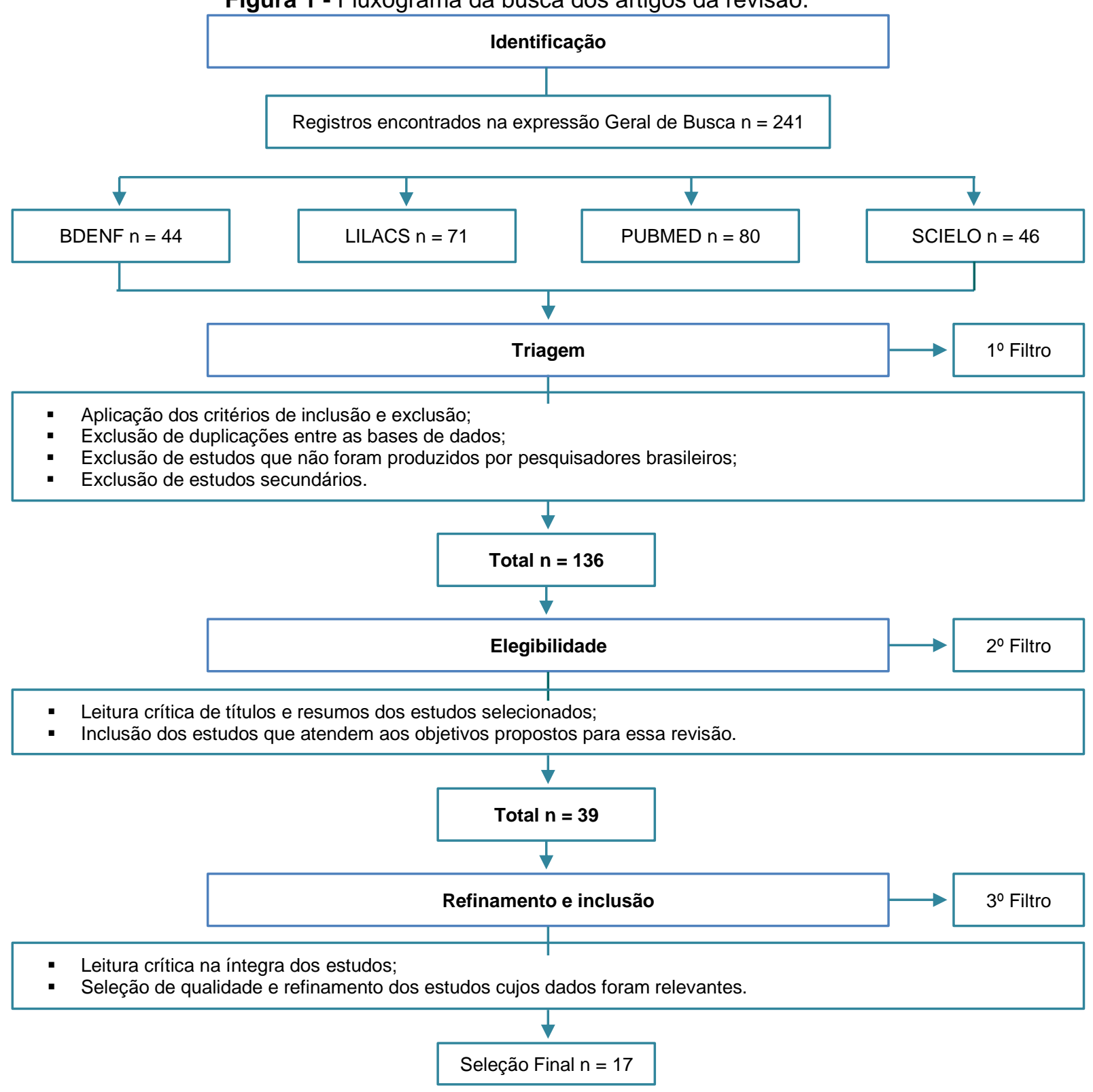

Fonte: Monteiro SA, et al., 2020.

Para organização e tabulação dos dados dos estudos selecionados, foi utilizado um protocolo de revisão integrativa para esse fim. Nesse protocolo se fez necessário identificar o título do estudo, sua instituição-sede, o local em que o trabalho foi publicado, as características metodológicas da pesquisa e a avaliação do rigor metodológico (SOUZA MT, et al., 2010).

Para realizar a classificação do nível de evidência dos trabalhos foi utilizada a categorização da Agency for Healthcare Research and Quality (AHRQ) (GALVÃO CM, 2006). A qualidade das evidências é classificada em seis níveis, a saber: Nível 1 - metanálise de múltiplos estudos controlados; Nível 2 - estudo individual com delineamento experimental; Nível 3 - estudo com delineamento quase-experimental como estudo sem randomização com grupo único pré e pós-teste, séries temporais ou caso-controle; Nível 4 - estudo com delineamento não-experimental como pesquisa descritiva correlacional e qualitativa ou estudos de caso; Nível 5 - relatório de casos ou dado obtido de forma sistemática, de qualidade verificável ou dados de avaliação de programas; Nível 6 - opinião de autoridades respeitáveis baseada na competência clínica ou opinião de comitê de especialistas, incluindo interpretações de informações não baseadas em pesquisas. 


\section{RESULTADOS}

Os cruzamentos de busca nas bases de dados estabelecidas resultaram na identificação de 44 artigos no banco de dados BDENF, 71 no LILACS, 80 na PUBMED e 16 na SciELO, totalizando 241 estudos. A aplicação dos três filtros possibilitou refinamento que resultou em seleção final de 17 artigos, com base na relevância e na qualidade dos dados para a presente revisão. Para melhor identificação de cada artigo selecionado, organizou-se uma exposição dos mesmos em sequência alfanumérica, iniciando em A1 até A17 (Quadro 1).

Quadro 1 - Estudos selecionados para análise.

\begin{tabular}{|c|c|c|c|c|c|}
\hline Código & Título do artigo & Autores & Ano & Periódico & $\begin{array}{l}\text { Base de } \\
\text { dados }\end{array}$ \\
\hline A1 & $\begin{array}{l}\text { Aplicativo informatizado com o } r \text { Nursing } \\
\text { Activities Score: instrumento para } \\
\text { gerenciamento da assistência em Unidade de } \\
\text { Terapia Intensiva. }\end{array}$ & $\begin{array}{l}\text { Castro MCN, } \\
\text { et al. }\end{array}$ & 2009 & $\begin{array}{l}\text { Texto \& Contexto } \\
\text { Enfermagem }\end{array}$ & LILACS \\
\hline $\mathrm{A} 2$ & $\begin{array}{l}\text { Carga de trabalho de enfermagem em Unidade } \\
\text { de Terapia Intensiva de Cardiologia e fatores } \\
\text { clínicos associados. }\end{array}$ & $\begin{array}{l}\text { Coelho FUA, } \\
\text { et al }\end{array}$ & 2011 & $\begin{array}{l}\text { Texto \& Contexto } \\
\text { Enfermagem }\end{array}$ & LILACS \\
\hline A3 & $\begin{array}{l}\text { Demanda de intervenções de enfermagem a } \\
\text { pacientes sob cuidados intensivos: NAS - } \\
\text { Nursing Activities Score. }\end{array}$ & $\begin{array}{l}\text { Feitosa MC, et } \\
\text { al. }\end{array}$ & 2012 & Escola Anna Nery & LILACS \\
\hline A4 & $\begin{array}{l}\text { Avaliação da carga de trabalho no pós- } \\
\text { operatório de cirurgia cardíaca segundo o } \\
\text { Nursing Activities Score. }\end{array}$ & $\begin{array}{l}\text { Oliveira LB, et } \\
\text { al. }\end{array}$ & 2015 & $\begin{array}{c}\text { Revista da Escola } \\
\text { de Enfermagem da } \\
\text { USP }\end{array}$ & LILACS \\
\hline A5 & $\begin{array}{l}\text { Carga de trabalho de enfermagem em Unidade } \\
\text { de Terapia Semi-intensiva especializada: } \\
\text { critérios para dimensionamento de pessoal. }\end{array}$ & $\begin{array}{l}\text { Trettene AS, } \\
\quad \text { et al. }\end{array}$ & 2015 & $\begin{array}{l}\text { Revista da Escola } \\
\text { de Enfermagem da } \\
\text { USP }\end{array}$ & MEDLINE \\
\hline A6 & $\begin{array}{l}\text { Nursing Activities Score: uma diretriz atualizada } \\
\text { para sua aplicação na Unidade de Terapia } \\
\text { Intensiva }\end{array}$ & $\begin{array}{l}\text { Padilha KG, et } \\
\text { al. }\end{array}$ & 2015 & $\begin{array}{c}\text { Revista da Escola } \\
\text { de Enfermagem da } \\
\text { USP } \\
\end{array}$ & LILACS \\
\hline A7 & $\begin{array}{l}\text { Padrão de intervenções de enfermagem } \\
\text { realizadas em vítimas de trauma segundo o } \\
\text { Nursing Activities Score. }\end{array}$ & $\begin{array}{l}\text { Nogueira LS, } \\
\text { et al. }\end{array}$ & 2015 & $\begin{array}{c}\text { Revista da Escola } \\
\text { de Enfermagem da } \\
\text { USP }\end{array}$ & LILACS \\
\hline A8 & $\begin{array}{l}\text { Carga de trabalho de enfermagem relacionada } \\
\text { ao índice de massa corporal de pacientes } \\
\text { críticos. }\end{array}$ & $\begin{array}{l}\text { Goulart LL, et } \\
\text { al. }\end{array}$ & 2017 & $\begin{array}{l}\text { Acta Paulista de } \\
\text { Enfermagem }\end{array}$ & LILACS \\
\hline A9 & Nursing Activities Score e a lesão renal aguda. & $\begin{array}{l}\text { Coelho FUA, } \\
\text { et al }\end{array}$ & 2017 & $\begin{array}{l}\text { Revista Brasileira } \\
\text { de Enfermagem }\end{array}$ & MEDLINE \\
\hline A10 & $\begin{array}{l}\text { Classificação de pacientes e carga de trabalho } \\
\text { de enfermagem em terapia intensiva: } \\
\text { comparação entre instrumentos. }\end{array}$ & $\begin{array}{l}\text { Ferreira PC, } \\
\quad \text { et al. }\end{array}$ & 2017 & $\begin{array}{l}\text { Revista Gaúcha de } \\
\text { Enfermagem }\end{array}$ & LILACS \\
\hline A11 & $\begin{array}{l}\text { Dimensionamento de pessoal de enfermagem } \\
\text { na UTI-Adulto de hospital universitário público. }\end{array}$ & Borges $\mathrm{F}$, et al & 2017 & $\begin{array}{c}\text { Cogitare } \\
\text { Enfermagem }\end{array}$ & LILACS \\
\hline A12 & $\begin{array}{l}\text { Dimensionamento de pessoal de enfermagem } \\
\text { referente à promoção do autocuidado em } \\
\text { unidade de terapia semi-intensiva pediátrica. }\end{array}$ & $\begin{array}{l}\text { Trettene AS, } \\
\text { et al. }\end{array}$ & 2017 & $\begin{array}{l}\text { Revista Brasileira } \\
\text { de Terapia } \\
\text { Intensiva } \\
\end{array}$ & MEDLINE \\
\hline A13 & $\begin{array}{l}\text { Dimensionamento do pessoal de enfermagem } \\
\text { na terapia intensiva adulto. }\end{array}$ & $\begin{array}{l}\text { Souza VS, et } \\
\text { al. }\end{array}$ & 2018 & $\begin{array}{l}\text { Revista Mineira de } \\
\text { Enfermagem }\end{array}$ & LILACS \\
\hline A14 & $\begin{array}{l}\text { Gravidade e carga de trabalho de enfermagem } \\
\text { em pacientes candidatos à vaga na UTI. }\end{array}$ & $\begin{array}{l}\text { Castro MCN, } \\
\text { et al. }\end{array}$ & 2018 & Escola Anna Nery & LILACS \\
\hline A15 & $\begin{array}{l}\text { Nursing Activities Score nos sítios assistenciais } \\
\text { em Unidade de Terapia Intensiva. }\end{array}$ & $\begin{array}{l}\text { Cyrino CMS, } \\
\quad \text { et al. }\end{array}$ & 2018 & Escola Anna Nery & LILACS \\
\hline A16 & $\begin{array}{l}\text { Associação entre carga de trabalho e } \\
\text { absenteísmo de profissionais de enfermagem } \\
\text { de nível médio. }\end{array}$ & $\begin{array}{c}\text { Feldhaus C, et } \\
\text { al. }\end{array}$ & 2019 & $\begin{array}{l}\text { Texto \& Contexto } \\
\text { Enfermagem }\end{array}$ & LILACS \\
\hline A17 & $\begin{array}{l}\text { Carga de trabalho de enfermagem e } \\
\text { mortalidade de pacientes na unidade de terapia } \\
\text { intensiva. }\end{array}$ & $\begin{array}{l}\text { Sobrinho } \\
\text { EDB, et al. }\end{array}$ & 2019 & $\begin{array}{l}\text { Revista Brasileira } \\
\text { de Ciências da } \\
\text { Saúde }\end{array}$ & LILACS \\
\hline
\end{tabular}

Fonte: Monteiro SA, et al., 2020. 
Dentre os 17 artigos (100\%) encontrados nessa produção, houve 5 artigos $(29,41 \%)$ publicados no ano de 2017, 4 artigos (23,53\%) publicados no ano de 2015, 3 artigos (17,65\%) publicados no ano de 2018, 2 artigos (11,77\%) publicados em 2019 e 1 artigo (5,88\%) publicado nos anos de 2009, 2011 e 2012.

No período de anos escolhido para a pesquisa, não foram encontradas publicações nos anos de 2010, 2013, 2014 e 2016. Identificou-se que a maior parte destes, no caso $15(88,23 \%)$ foi publicada em revistas científicas na área de Enfermagem, e apenas $2(11,77 \%)$ em revistas multidisciplinares, Revista Brasileira de Terapia Intensiva e Escola Brasileira de Ciências da Saúde.

Dois periódicos, Texto e Contexto Enfermagem e Escola Anna Nery, publicaram três artigos (17,65\%) sobre o tema foco, um periódico, Revista da Escola de Enfermagem da USP, publicou quatro artigos (23,53\%), sendo que os demais, Acta Paulista de Enfermagem, Revista Brasileira de Enfermagem, Revista Gaúcha de Enfermagem, Cogitare Enfermagem, Revista Brasileira de Terapia Intensiva, Revista Mineira de Enfermagem e Revista Brasileira de Ciências da Saúde, publicaram um (5,88\%).

Nesse contexto, realizou-se a classificação dos artigos conforme o nível de evidência por meio da categorização da AHRQ (GALVÃO CM, 2006), onde se observou que 16 artigos estão inseridos no nível de evidência 5: relatório de casos ou dado obtido de forma sistemática, de qualidade verificável ou dados de avaliação de programas e 1 artigo no nível de evidência 4: estudo com delineamento não-experimental como pesquisa descritiva correlacional e qualitativa ou estudos de caso.

No que tange a abordagem de pesquisa, os 16 estudos (94,12\%) são de cunho quantitativo e 1 (5,88\%) de cunho qualitativo. Quanto ao tipo, há uma predominância de $05(29,41 \%)$ estudos descritivos, enquanto 5 $(29,41 \%)$ realizaram estudo transversal, $04(23,53 \%)$ caracteriza-se como coorte, 1 (5,88\%) pesquisa multicêntrica e $1(5,88 \%)$ estudo prospectivo.

Para a realização da caracterização da abordagem de conteúdo das publicações identificadas para a revisão, construiu-se a Tabela 2, onde foi possível a realização da apresentação das seguintes variáveis: ordem, objetivo, tipo de estudo, abordagem, principais resultados e conclusão.

No que diz respeito a matriz do assunto foco, é abordado a aplicação do Nursing Activities Score (NAS) na realização da mensuração da carga de trabalho de enfermagem em UTI's pediátricas, renais, cardíacas, de trauma, dimensionamento de pessoal, discute sobre a trajetória da criação de um aplicativo que apresenta o conteúdo do Nursing Activities Score (NAS), é comparado com a Resolução 293/04 e o score SAPES-3 de pacientes internados nas Unidade de Terapia Intensiva (UTI's). 
Quadro 2 - Caracterização dos artigos selecionados para análise segundo ordem, objetivo, tipo e abordagem do estudo.

\begin{tabular}{|c|c|c|c|}
\hline Código & Objetivo & $\begin{array}{l}\text { Tipo de } \\
\text { estudo }\end{array}$ & $\begin{array}{l}\text { Abordagem } \\
\text { do estudo }\end{array}$ \\
\hline A1 & $\begin{array}{l}\text { Demonstrar a trajetória da construção de um aplicativo (software) com o } \\
\text { conteúdo do Nursing Activities Score e suas características operacionais. }\end{array}$ & Descritivo & Qualitativa \\
\hline $\mathrm{A} 2$ & $\begin{array}{c}\text { Identificar a carga de trabalho de enfermagem em Unidade de Terapia } \\
\text { Intensiva de Cardiologia e verificar a associação dessa variável } \\
\text { com características demográficas e clínicas dos pacientes. }\end{array}$ & $\begin{array}{c}\text { Coorte } \\
\text { Retrospectivo }\end{array}$ & Quantitativa \\
\hline A3 & $\begin{array}{l}\text { Avaliar a demanda de cuidados de enfermagem pela aplicação do Nursing } \\
\text { Activities Score (NAS). }\end{array}$ & Descritivo & Quantitativa \\
\hline A4 & $\begin{array}{l}\text { Identificar fatores associados à carga de trabalho dos cuidados de } \\
\text { enfermagem para pacientes no período pós-operatório de cirurgia cardíaca. }\end{array}$ & $\begin{array}{l}\text { Coorte } \\
\text { prospectivo }\end{array}$ & Quantitativa \\
\hline A5 & $\begin{array}{l}\text { Avaliar a carga de trabalho de enfermagem na Unidade de Terapia Semi- } \\
\text { intensiva, especializada no atendimento de crianças com anomalias } \\
\text { craniofaciais e síndromes associadas; comparar a quantidade de mão-de- } \\
\text { obra necessária de acordo com o Nursing Activities Score e a Resolução } \\
\text { COFEN 293/04. }\end{array}$ & $\begin{array}{l}\text { Descritivo } \\
\text { Transversal }\end{array}$ & Quantitativa \\
\hline A6 & $\begin{array}{l}\text { Descrever a carga de trabalho de enfermagem em Unidades de Terapia } \\
\text { Intensiva de diferentes países, de acordo com os escores obtidos no Nursing } \\
\text { Activities Score e verificar a concordância entre os países quanto à } \\
\text { interpretação das diretrizes do NAS. }\end{array}$ & $\begin{array}{l}\text { Multicêntrico } \\
\text { transversal }\end{array}$ & Quantitativa \\
\hline A7 & $\begin{array}{l}\text { Identificar o padrão das intervenções de enfermagem realizadas em vítimas } \\
\text { de trauma na Unidade de Terapia Intensiva. }\end{array}$ & $\begin{array}{l}\text { Transversal } \\
\text { Prospectiva }\end{array}$ & Quantitativa \\
\hline A8 & $\begin{array}{l}\text { Mensurar e comparar a carga de trabalho de enfermagem e a frequência } \\
\text { dos itens pontuados no Nursing Activities Score, considerando os diferentes } \\
\text { grupos de IMC de pacientes internados em Unidade de Terapia Intensiva. }\end{array}$ & $\begin{array}{l}\text { Descritivo } \\
\text { Longitudinal }\end{array}$ & Quantitativa \\
\hline A9 & $\begin{array}{l}\text { Avaliar a carga de trabalho de enfermagem em pacientes de terapia } \\
\text { intensiva com lesão renal aguda (LRA). }\end{array}$ & $\begin{array}{c}\text { Coorte } \\
\text { Retrospectivo }\end{array}$ & Quantitativa \\
\hline A10 & $\begin{array}{l}\text { Avaliar as médias da carga de trabalho de enfermagem obtidas por meio do } \\
\text { Nursing Activities Score, bem como os extratos do grau de dependência de } \\
\text { pacientes obtidos pelo Sistema de Classificação de Pacientes de Perroca. }\end{array}$ & Prospectivo & Quantitativa \\
\hline A11 & $\begin{array}{l}\text { Dimensionar o quadro de pessoal de enfermagem de uma Unidade de } \\
\text { Terapia Intensiva para adultos do Paraná e compará-lo com o quadro real } \\
\text { existente. }\end{array}$ & $\begin{array}{l}\text { Transversal- } \\
\text { documental }\end{array}$ & Quantitativa \\
\hline A12 & $\begin{array}{l}\text { Calcular e comparar o dimensionamento de pessoal no que diz respeito à } \\
\text { promoção do autocuidado em unidade de terapia semi-intensiva pediátrica. }\end{array}$ & Prospectivo & Quantitativa \\
\hline A13 & $\begin{array}{l}\text { Dimensionar o quadro de pessoal de enfermagem de uma unidade de } \\
\text { terapia intensiva para adultos. }\end{array}$ & $\begin{array}{l}\text { Transversal } \\
\text { Prospectivo } \\
\text { Descritivo } \\
\end{array}$ & Quantitativa \\
\hline A14 & $\begin{array}{l}\text { Identificar a gravidade e a carga de trabalho de enfermagem requerida por } \\
\text { pacientes adultos candidatos à vaga em Unidade de Terapia Intensiva. }\end{array}$ & $\begin{array}{l}\text { Transversal } \\
\text { Exploratório- } \\
\text { descritivo } \\
\text { Prospectivo }\end{array}$ & Quantitativa \\
\hline A15 & $\begin{array}{l}\text { Comparar o Nursing Activities Score (NAS) entre os Sítios Assistenciais na } \\
\text { Unidade de Terapia Intensiva. }\end{array}$ & $\begin{array}{c}\text { Descritivo } \\
\text { Retrospectivo }\end{array}$ & Quantitativa \\
\hline A16 & $\begin{array}{l}\text { Identificar o absenteísmo da equipe de enfermagem de nível médio em uma } \\
\text { Unidade de Terapia Intensiva e verificar a associação entre absenteísmo e } \\
\text { carga de trabalho. }\end{array}$ & $\begin{array}{l}\text { Transversal } \\
\text { Retrospectivo } \\
\text { Documental }\end{array}$ & Quantitativa \\
\hline A17 & $\begin{array}{l}\text { Analisar a correlação entre a carga de trabalho da equipe de enfermagem, } \\
\text { aferida pelo Nursing Activities Score e a mortalidade predita pelo escore } \\
\text { SAPS } 3 \text { dos pacientes internados em Unidade de Terapia Intensiva, } \\
\text { verificando as correlações entre os profissionais de enfermagem atuantes } \\
\text { na unidade segundo o preconizado pela RDC e o recomendado pelo escore } \\
\text { NAS. }\end{array}$ & Coorte & Quantitativa \\
\hline
\end{tabular}

Fonte: Monteiro SA, et al., 2020. 


\section{DISCUSSÃO}

No Brasil, foi observado uma fragilidade na universalidade dos serviços do Sistema Único de Saúde (SUS), principalmente os de alta complexidade e alto custo, como as internações em leitos de UTI. Existem 17.940 leitos de UTI credenciados, sendo que 11.615 são destinados a pacientes adultos, número este que não supre as demandas existentes. Com isso, o acesso acaba se tornando limitado e o atendimento aos pacientes graves, tardio, podendo levar ao comprometimento dos resultados clínicos e da taxa de mortalidade (CASTRO MCN, et al., 2018).

No estudo de Castro MCS, et al. (2018), os pacientes foram divididos em dois grupos: pacientes admitidos e não admitidos, sendo eles 75 e 85 respectivamente, tendo em vista que 11 evoluíram a óbito antes da resposta do pedido à vaga na UTI e 8 pacientes tiveram o pedido cancelado antes da decisão da equipe. Sendo o NAS de $63,09 \%$ para os pacientes admitidos e $53,85 \%$ para os pacientes não admitidos, enquanto os pacientes que evoluíram a óbito o escorem NAS foi de $96,9 \%$.

Ainda que o dimensionamento de pessoal não faça parte da finalidade do NAS, é possível compreender como um instrumento que vem integrar e colaborador o planejamento em recursos humanos. Com isso, observa-se que o NAS vem sendo o principal instrumento utilizado para mensurar a carga de trabalho da equipe de enfermagem em UTI's brasileiras e nos demais países, tornando-se uma ferramenta segura de avaliação e de gestão, auxiliando os enfermeiros no dimensionamento adequado da equipe de enfermagem e na consonância das práticas seguras de cuidado (CASTRO MCN, et al., 2018).

Observou-se pelo estudo de Trettene AS, et al. (2017) que ao se comparar a carga de trabalho de enfermagem em UTI pediátrica segundo as horas do NAS e a resolução do COFEN 527/16, o requerido, segundo o NAS, foi superior e a carga de trabalho em enfermagem foi avaliada em primeira e segunda internação. Em relação a carga de trabalho de enfermagem referente à primeira internação, a média foi de $60,9 \%$, enquanto a segunda internação foi de $41,6 \%$, com uma diferença de $19,3 \%$, considerando também um total de 14,9 horas na primeira internação e 9,9 horas na segunda.

Ademais, Sobrinho EDB, et al. (2019) afirmam que, quando analisado o dimensionamento de enfermagem das unidades incluídas, o mesmo estava próximo do indicado pelo NAS em números de profissionais de enfermagem. Quando realizou a análise dos dispostos na Resolução COFEN no 293/2004, observou-se que o número de enfermeiros era menor que o recomendado para essa demanda assistencial, pois, de acordo com essa resolução, é necessário pelo menos $54 \%$ do corpo funcional de profissionais com nível superior.

Foi destacado também que a unidade possuía o dimensionamento mínimo de enfermagem recomendado pela RDC № 7 e RDC ํo 26, que preconiza a existência de 1 técnico de enfermagem para cada 2 leitos ou fração e 1 enfermeiro para cada 10 leitos, o que representa, de maneira preliminar, a fragilidade em realizar um dimensionamento de enfermagem apenas pelas regras mínimas da legislação vigente no Brasil (SOBRINHO EDB, et al., 2019).

Nesse contexto, Ferreira PC, et al. (2017) fazem um comparativo entre o NAS e o Sistema de Classificação de Pacientes (SCP), mensurando nas primeiras $24 \mathrm{~h}$ a carga de trabalho de enfermagem de acordo com o grau de dependência dos pacientes. A média do NAS obteve uma diferença de 7,3 horas do SCP, o que implica uma elevada carga de trabalho de enfermagem. A pontuação do NAS se concentrou no domínio de "atividades básicas" enquanto no SCP, o destaque foi em "cuidado corporal e eliminações", "cuidado com a pele e mucosas" e "locomoção e atividade". Dessa forma, todas as áreas de cuidados do SCP são contempladas dentro dos domínios do NAS.

Borges F, et al. (2017) utilizaram o NAS junto à Resolução do COFEN 543/17, onde a variação do NAS foi de 1258,6 a 2259,0 pontos com dispersão esperada e o dimensionamento de pessoal de 500,2, considerando as 5 equipes disponíveis e o acrescimento de Índice de Segurança Técnico em 15\%, onde caberia 87 trabalhadores. Contudo a unidade contava com 69 profissionais, sendo 11 enfermeiros e 58 técnicos de enfermagem, porém 9 dos técnicos se encontravam afastados, obtendo um total de 60 profissionais. 
Segundo Nogueira LS, et al. (2015), o NAS é utilizado para mensurar a carga de trabalho de enfermagem pelo perfil sociodemográfico de vítimas de trauma admitidas nas primeiras $24 \mathrm{~h}$, e identificou-se que a variação foi de $35,9 \%$ a $131,5 \%$, com predominância do sexo masculino. Os itens mais pontuados foram: atividades de monitoração e controle, investigações laboratoriais, medicação, exceto drogas vasoativas, procedimentos de higiene, mobilização e posicionamento, suporte e cuidados aos familiares e pacientes, tarefas administrativas e gerenciais, e medida quantitativa de débito urinário.

Cyrino CMS, et al. (2018) realizaram a comparação da carga de trabalho de enfermagem sendo mensurada pelo NAS nos diversos sítios assistenciais em uma UTI, com prevalência do sexo masculino. No entanto, na comparação do estudo Feitosa MC, et al. (2012) quanto a variável sexo, evidenciaram uma predominância de pacientes do sexo feminino $(64,4 \%)$, visto que poucos estudos trazem esse relato em relação ao número de mulheres. Os pacientes foram divididos em sítio pós-operatório, sítio de isolamento e sítio de longa permanência. A maior média do NAS aconteceu no sítio do pós-operatório em setembro, seguida pelo sítio de isolamento em agosto, sendo que, a carga de trabalho de enfermagem permaneceu constante entre os meses.

Dessa forma, dependendo do sítio assistencial, o NAS pode organizar o processo de trabalho na UTI e adequar o dimensionamento da equipe de enfermagem, considerando as diferentes características dos pacientes, contribuindo para a qualidade dos cuidados e a segurança do paciente, uma vez que, os pacientes que permanecem maior tempo em cuidados intensivos, são, provavelmente, aqueles mais instáveis e clinicamente mais graves (OLIVEIRA LB, et al., 2015; CYRINO CMS, et al., 2018).

No que diz respeito a vertente idade, foi observado o domínio de duas faixas etárias, a saber, 58 a 77 anos $(37,8 \%)$, seguida de 18 a 37 anos (35,5\%). Quanto ao tempo de internação, a média foi de sete dias, sendo que a maioria $(66,7 \%)$ permaneceu internada de dois a oito dias, e os motivos das internações foram de $35,5 \%$ por pós- operatório imediato, $28,9 \%$ por doenças cardiovasculares e $20,0 \%$ por doenças respiratórias, sendo diretamente proporcionais as faixas etárias que predominam no estudo. Dessa forma, o percentual do NAS variou de $39,2 \%$ a $133,7 \%$, sendo a média total de $67,3 \%$, que foi compatível com estudos nacionais que variaram de $61,9 \%$ a $73,7 \%$ (FEITOSA MC, et al., 2012).

No contexto da especialidade cardiológica Oliveira LB, et al. (2015) evidenciaram que o NAS de 24 horas pode corresponder a 19,7 horas de trabalho da equipe de enfermagem por paciente. Coelho FUA, et al. (2017) realizaram uma comparação com a carga de trabalho mensurada pelo NAS com outras variáveis, sendo elas: Pontuação Simplificada de Fisiologia Aguda 2 (SAPS II) e Índice de Disfunção Orgânica (LODS), e relatou que o NAS foi em média $66,6 \%$ maior frente aos demais, em um turno de 8 horas na UTI cardiológica. Pela pontuação do NAS, constatou-se que o valor total de pessoal de enfermagem necessário era de 32, sendo 17 enfermeiros e 15 técnicos, o que divergia do cenário observado (SOUZA VS, et al., 2018).

De acordo com Coelho FUA, et al., (2017) a Lesão Renal Aguda (LRA) é uma das condições mais graves em pacientes hospitalizados relacionados ao grande impacto de suas complicações e quando estão associados a pacientes críticos, os desfechos podem ainda ser mais agressivos. Com isso, o instrumento foi usado para mensurar a carga de enfermagem em pacientes com LRA. Houve maior pontuação em pacientes com LRA com os que não a desenvolveram. A média do NAS foi de $42,2 \%$, semelhantes aos pacientes de estágio 1 e 2, sem mudanças significativas, porém 35 pacientes de estágio 2 com LRA tiveram pontuação do NAS bem superior aos pacientes sem LRA, enquanto que os indivíduos de estágio 3 apresentaram maior pontuação em comparação aos pacientes sem LRA e de estágio 1 e 2.

No estudo de Goulart LL, et al, (2017) os pacientes foram divididos em 3 grupos de acordo com seu IMC: baixo peso $(6,4 \%)$, peso normal e pré-obeso $(73,7 \%)$ e obeso $(19,9 \%)$. Com isso, foi observado que os valores médios mensurados pelo NAS não obteve diferenças significativas entre os grupos, tanto na admissão quanto na saída. O score da admissão foi utilizado para definir a carga horária de enfermagem de acordo com IMC do paciente, assim definindo suas intervenções. Os itens de procedimentos de higiene, mobilização e posicionamento tiveram maior porcentagem nos pacientes obesos, onde a carga de trabalho mensurada foi semelhante à observada em outras UTI's, ou seja, entre 62,2 e $70,4 \%$. 
Feldhaus C, et al. (2019) fizeram mensurações do score NAS para verificar se há uma comparação entre absenteísmo e a carga de trabalho entre duas equipes (UTI I e UTI II), onde as longas jornadas de trabalho, horas extras e pluralidade das funções, fragilizavam o serviço e a qualidade da assistência a ser prestada. Foram prestadas 4.920 e registradas um total de 461 abstenções, onde o seguimento da UTI II teve maior proporção e ausências comparadas a da UTI I. Dessa forma, o NAS médio foi maior na UTI II, uma vez que os valores se mostraram mais elevados do que na UTI I.

O NAS, além de mensurar a carga de trabalho da enfermagem e o quantitativo de pessoal, se apresenta como um instrumento promissor para avaliar o índice de gravidade do paciente, podendo ser implementado em todo o mundo. Para tanto, foi realizado um comparativo da carga de trabalho em enfermagem mensurada pelo NAS e dúvidas na pontuação dos itens do instrumento em um estudo multicêntrico que envolveu Noruega, Holanda, Polônia, Espanha, Egito, Grécia e Brasil, cujo objetivo foi padronizar um consenso de uma só versão atualizada do instrumento (PADILHA KG, et al., 2015).

Atualmente, os instrumentos que são mais utilizados na UTI para avaliar a gravidade do paciente são o Acute Physiologic and Chronic Health Evaluation (APACHE) e o Simplified Acute Physiologic Score (SAPS). Entretanto, não são capazes de avaliar a carga de trabalho da enfermagem, uma vez que são direcionados à condição clínica do paciente e à terapêutica médica. Dessa forma, Castro MCN, et al. (2009) desenvolveram um aplicativo móvel com base no score NAS, onde o enfermeiro coleta os dados e avalia o paciente de acordo com a demanda dos cuidados de enfermagem, e o app armazena as informações e calcula a pontuação obtida, compartilhando os dados através de webservice.

\section{CONSIDERAÇÕES FINAIS}

Observou-se que o NAS tem como finalidade avaliar o paciente diante de suas necessidades, para que sejam calculadas quantas horas de cuidados de enfermagem são necessários para supri-las. Também é utilizada nas Unidades de Terapia Intensiva brasileiras de forma indireta, como instrumento auxiliar na mensuração da carga de trabalho da equipe de enfermagem, quantificando a carga horária dos cuidados prestados, refletindo diretamente na evolução clínica do paciente considerado crítico, e em diferentes setores da instituição hospitalar. Quanto a porcentagem em horas mensuradas, na qual implica no número de profissionais necessários para a assistência dentre das UTI's, verificou-se dissonância com outros instrumentos, normas técnicas e resoluções vigentes, havendo um número de profissionais inferior ao requerido pelo NAS. Portanto, evidencia-se um novo campo para futuras pesquisas quanto ao manuseio do NAS nos demais setores hospitalares, aqueles considerados não críticos para a assistência.

\section{REFERÊNCIAS}

1. BACKES DS, et al. O papel profissional do enfermeiro no Sistema Único de Saúde: da saúde comunitária à estratégia de saúde da família. Ciênc. Saúde Coletiva, 2012; 17(1): 223-230.

2. BORGES $\mathrm{F}$, et al. Dimensionamento de pessoal de enfermagem na uti-adulto de hospital universitário público. Cogitare Enfermagem, 2017; 22(2): e50306.

3. BRASIL. Ministério da Saúde. Diretrizes e normas regulamentadoras de pesquisa envolvendo seres humanos. Brasília: Ministério da Saúde; 2012.

4. BRASIL. Ministério da Saúde. Diretrizes e normas regulamentadoras de pesquisa envolvendo seres humanos. Brasília: Ministério da Saúde; 2016.

5. CASTRO MCN, et al. Aplicativo informatizado com o Nursing Activities Score: Instrumento para gerenciamento da assistência em Unidade de Terapia Intensiva. Texto Cont Enferm, 2009; 18(3): 577-85.

6. CASTRO MCN, et al. Gravidade e carga de trabalho de enfermagem em pacientes candidatos à vaga na UTI. Esc Anna Nery, 2018; 22(1): e20170167.

7. COELHO FUA, et al. Carga de trabalho de enfermagem em unidade de terapia intensiva de cardiologia e fatores clínicos associados. Texto Cont Enferm, 2017; 20(4): 735-41.

8. COELHO FUA, et al. Nursing Activities Score and acute kidney injury. Rev Bras Enferm, 2017; 70(3): 475-480.

9. CYRINO CMS, et al. Nursing Activities Score nos sítios assistenciais em Unidade de Terapia Intensiva. Esc Anna Nery, 2018; 22(1): e20170145. 
10. FEITOSA MC, et al. Demanda de intervenções de enfermagem a pacientes sob cuidados intensos: NAS- Nursing Activites Score. Esc Anna Nery, 2012; 16(4): 682-688.

11. FELDHAUS C, et al. Associação entre a carga de trabalho e absenteísmo de profissionais de enfermagem no nível médio. Texto Cont Enferm, 2019; 28(esp): e20180307.

12. FERREIRA PC, et al. Classificação de pacientes e carga de trabalho de enfermagem em terapia intensiva: comparação entre instrumentos. Rev Gaúcha Enferm, 2017; 38(2): e62782.

13. GALVÃO CM. Níveis de evidência. Acta Paul Enferm, 2006; 19(2).

14. GOULART LL, et al. Carga de trabalho de enfermagem relacionada ao índice de massa corporal de pacientes críticos. Acta Paul Enferm, 2017; 30(1): 31-38.

15. NOGUEIRA LS, et al. Padrão de intervenções de enfermagem realizadas em vítimas de trauma segundo o Nursing Activities Score. Rev Esc Enferm USP, 2015; 49(esp): 29-35.

16. NOVARETTI MCZ, et al. Gestão em Unidades de Terapia Intensiva brasileira: estudo bibliométrico dos últimos 10 anos. Rahis, 2015; 12(4): 16-33.

17. OLIVEIRA LBD, et al. Avaliação da carga de trabalho no pós-operatório de cirurgia cardíaca segundo o Nursing Activities Score. Rev Esc Enferm USP, 2015; 49(esp): 80-86.

18. PADILHA KG, et al. Nursing activities score: uma diretriz atualizada para sua aplicação na Unidade de Terapia Intensiva. Rev Esc Enferm USP, 2015; 49(esp): 131-137.

19. POLIT DF, BECK CT. Fundamentos de Pesquisa em enfermagem: avaliação de evidências para as práticas da enfermagem. 9nd ed. Porto Alegre: Artmed, 2018; 456p.

20. SOBRINHO EDB, et al. Carga de trabalho da enfermagem e a mortalidade de pacientes nas unidades de terapia intensiva. Rev Bras de Ciência da Saúde, 2019; 23(3): 297-308.

21. SOUZA MT, et al. Revisão integrativa: o que é e como fazer. Einstein, 2010; 8(1): 102-106.

22. SOUZA VS, et al. Dimensionamento do pessoal de enfermagem na terapia intensiva adulto. Rev Min Enferm, 2018; 22(esp.): e-1121.

23. TRETTENE AS, et al. Carga de trabalho de enfermagem em unidade de terapia semi-intensiva especializada: critérios de tamanho da força de trabalho. Rev Esc Enferm USP, 2015: 49(6): 958-963.

24. TRETTENE AS, et al. Dimensionamento de pessoal de enfermagem referente à promoção do autocuidado em unidade de terapia semi-intensiva pediátrica. Rev Bras Ter Intensiva, 2017; 29(2): 171-179.

25. VERSA GIGS, et al. Influência do dimensionamento da equipe de enfermagem na qualidade do cuidado ao paciente crítico. Texto \& Contexto Enfermagem, 2011; 20(4): 796-802. 